\title{
CFD Study of Aquatic Thrust Generation by an Octopus-like Arm
} Under Intense Prescribed Deformations

\author{
Asimina Kazakidi ${ }^{1 \mathrm{a}}$, Dimitris P. Tsakiris ${ }^{\mathrm{a}}$, Dionysios Angelidis ${ }^{\mathrm{b}}$, Fotis Sotiropoulos $^{\mathrm{b}}$, \\ John A. Ekaterinaris ${ }^{c, d}$ \\ ${ }^{a}$ Institute of Computer Science, Foundation for Research E Technology - Hellas (FORTH), 70013 Heraklion, Crete, Greece \\ ${ }^{b}$ St. Anthony Falls Laboratory, University of Minnesota, Minneapolis, MN, 55455-0116, USA \\ ${ }^{c}$ Department of Aerospace Engineering, Embry-Riddle Aeronautical University, Daytona Beach, FL, 32114, USA \\ ${ }^{d}$ Institute of Applied $\mathcal{E}$ Computational Mathematics, FORTH, 70013 Heraklion, Crete, Greece
}

\begin{abstract}
The complexity in structure and locomotion of cephalopods, such as the octopus, poses difficulties in modeling and simulation. Their slender arms, being highly agile and dexterous, often involve intense deformations, which are hard to simulate accurately, while simultaneously ensuring numerical stability and low diffusion of the transient motion results. Within the Immersed-Boundary framework, this paper focuses on an arm geometry performing prescribed motions that reflect octopus locomotion. The method is compared with a Finite-Volume numerical approach to determine the mesh requirements that must be employed for sufficiently capturing, not only the near wall viscous flow, but also the off-body vortical flow field in intense forced motions. The objective is to demonstrate and exploit the generality of the immersed boundary approach to complex numerical simulations of deforming geometries. Incorporation of arm deformation was found to increase the output thrust of a single-arm system. It was further found that sculling motion combined with arm undulations provides an effective propulsive scheme for an octopus-like arm.
\end{abstract}

Keywords: Computational fluid dynamics (CFD), aquatic locomotion, biological propulsion, large deformations, immersed boundary method

\footnotetext{
${ }^{1}$ Corresponding author: Email: kazakidi@ics.forth.gr; Tel.: +30 281q 391695; Fax: +30 2810391601.
} 


\section{Introduction}

The appendages of cephalopods (i.e., the arms and tentacles of squids and cuttlefish, the octopus arms, and the nautilus tentacles) are elongated muscular structures, composed of a tight 3D musculature maintaining constant volume despite large deformations (termed muscular hydrostats [1]). Cephalopods may use their arms and, for squids and cuttlefish, also their fins, to perform a large repertoire of movements and behaviors [2,3]. We are interested in investigating the individual role of octopus arms in aquatic locomotion and the generated hydrodynamic forces, which have not been explored in detail. Studies of fish swimming (as well as of bird flight) have shown that various marine animals, as well as birds, are able to exploit and control perturbations found in the surrounding fluid (e.g. vortices and eddies), in order to limit the levels of energy consumption and augment the produced thrust $[4,5,6,7,8,9,10,11,12,13,14]$. This work aims to investigate the flow dynamics around intense octopus-like motions, in an attempt to shed light on the underlying mechanisms and the energetics of cephalopod swimming.

The main difficulty in numerical flow simulations around time-varying geometries involving intense motions, is that the computational grid must deform over time, following the motion of the body (body-fitted methods). Finite volume (FV) body conforming methods include mesh deformation techniques that fulfill the geometric conservation requirement $[15,16]$ for small deformations and can provide high-fidelity results. These methods have the advantage of capturing in great detail the boundary layer around the swimming body and can simulate flows at high Reynolds numbers. They also allow for a direct application of the boundary conditions on the moving body. However, the mesh generation is demanding and the computational cost, for methods involving moving mesh or re-meshing strategies at every time step during a simulation, can be significantly high. For large grid deformations, in particular, re-meshing may affect the stability and accuracy of the numerical scheme [15].

Fixed-grid numerical methods, on the other hand, have attracted much attention in recent years, particularly for complex fluid-structure interaction problems. The advantage of these methods is their efficiency in handling arbitrarily large deformations with relatively low computational cost, since no mesh-updating strategies are involved. Instead, the effect of the moving body is defined on a fixed computational domain through the addition of external forces in the governing equations of motion. The external force field must satisfy the no-slip condition of the fluid on the moving solid body. Some of the challenges that fixed-grid methods face are related to the accurate definition of the 
48 moving body with respect to the fixed domain, and the successful application of the boundary conditions. The best-

49 known fixed-grid numerical method for moving bodies is the immersed boundary method (IBM) of Peskin (1972)

$[17,18]$, where a delta function is implemented for the distribution of the surface forces over several mesh nodes around the moving body, in order to avoid numerical instabilities. This produces smearing of the interface and, hence, immersed boundary methods require increased spatial resolution around the moving body. Further developments in this approach are limited to solutions with moderate Reynolds numbers [19, 20]. Approaches that overcome these (HCIB) $[22,23,24,25]$.

Here, we focused on the use of an immersed-boundary HCIB approach $[24,25]$ to implement and study prescribed

deformations, because of its robustness and handling capability. The mesh requirements for capturing the boundary layer and evaluate accurately the generated hydrodynamic forces were determined by comparing the method with a finite-volume approach $[26,27]$. This investigation lies within a well-known field of research on aquatic animal locomotion, on which there exists a considerable amount of literature $[4,5,6,7,8,9,10,11,12,13,14,28]$. Nevertheless, many questions on aquatic swimming remain unanswered, in particular for cephalopods. This work seeks to study the flow dynamics around intense octopus-like motions, utilizing computational fluid dynamic techniques on time-varying arm geometries performing prescribed motions. It also aims to demonstrate and exploit the generality of the immersed boundary approach to complex numerical simulations of such geometries. Incorporation of arm deformation was found to increase the output thrust of a single-arm system. It was further found that sculling motion combined with arm undulation is an effective propulsive scheme for an octopus-like arm.

The remainder of the paper is organized as follows: Section 2 discusses the arm kinematics and the numerical frameworks utilized; Section 3 presents flow simulations around an arm with and without the effect of prescribed deformation; Section 4 summarizes the scopes of this study and concludes with suggestions for further work. 

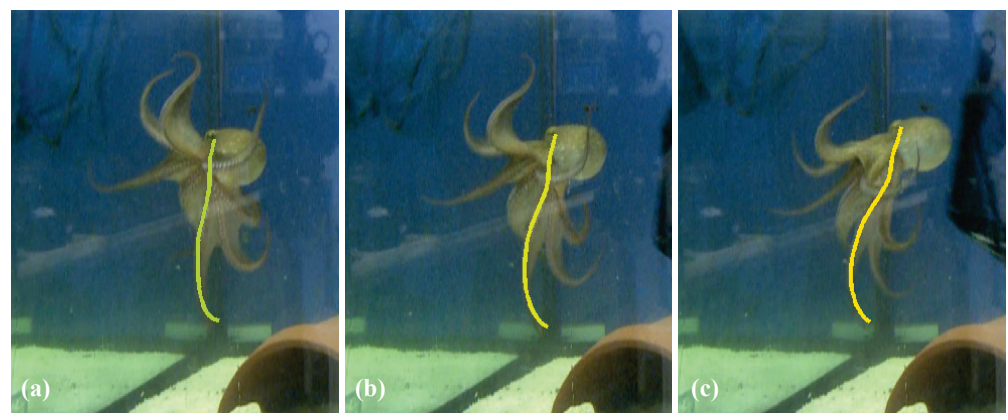

(d)

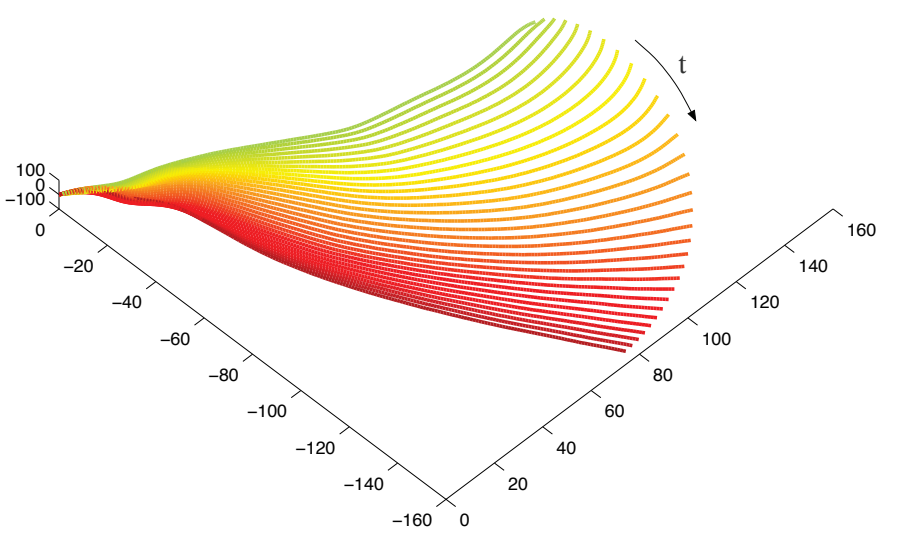

Figure 1: (a-c) Snapshots of octopus swimming in an aquarium [29]. The images are superimposed with segmented midlines of a single arm, color-coded according to the 3D arm reconstruction of (d), following the process described in the text. (d) The 3D arm trajectories are plotted here after translation of the starting point (here the eye of the octopus) to zero [30, 31, 32]; all dimensions are in millimetres. The plot concerns only the power stroke part (closing of the arms) of a more-complicated arm-swimming motion.

\section{Methods}

\subsection{Octopus arm kinematics}

Figures 1a-1c show snapshots of an octopus performing arm-swimming motion in an aquarium [29]. The images also depict the topological skeleton (midline) of a single arm, after being extracted automatically, for every snapshot, following the process described in detail in Yekutieli et al. [33]. Such 2D midlines can be extracted, similarly, from multiple cameras, synchronized to record the motion of live octopus. Using a set of three high-definition cameras and a checkerboard pattern for camera calibration [30,31], the intrinsic and extrinsic camera parameters were estimated, according to a traditional grid based calibration technique [34]. This process allows for a robust calculation of the world Cartesian coordinates from the projective pinhole camera coordinates. By employing epipolar geometry con- 
straints and least-square approximation, between each camera view, the 3D reconstruction of the medial axis of an individual arm was obtained for every video frame [31,32]. Figure 1d displays the reconstructed 3D trajectories of the arm indicated in Figures 1a-1c; the trajectories are plotted after translation, such that their starting point (corresponding, here, to the eye of the octopus) is at zero position at all times. This allows identification of the arm motion separately from the motion of the animal's body. The motion of the arm during swimming is evidently complex and, therefore, such motions are in general difficult to model. However, the motion can be simplified and be broadly characterized by a primary rotation around the starting position, and a deformation with time. Such approach is useful in capturing the fundamental characteristics of complex movements and was also used in this work.

\subsection{Arm geometry and motion approximation}

A first attempt to model realistic movements of individual cephalopod appendages, such as the reconstructed trajectories shown in Figure 1, is to consider simplified rotations of a slender octopus-like arm, around its fixed base. The arm was approximated with a right circular conical frustum, of a 9.75:1 taper ratio (length, L, to base-diameter, D) and a 17.5:1 aspect ratio (length to mean-diameter), for numerical simulations in quiescent fluid (Figures 2, 3). The temporal variation of the angular velocity $\omega(t)$ and the angle of rotation $\phi(t)$ may take various forms for a two-stroke motion profile, in which the arm rotates upwards and downwards in a cyclic way. Here, we examine two basic profiles, as displayed in Figures 2a, 2b: a sinusoidal oscillation, and a sculling profile $[35,36,37,38]$ of different velocity ratio $\beta$ between a relatively slow upstroke (termed as recovery stroke [35]) and a considerably faster downstroke (termed power stroke [35]). These motion profiles originate from observations of live octopus and analysis of the reconstructed 3D arm trajectories, during arm-swimming motion, in the way described in the previous paragraph and presented in Figure 1. The profiles can be formulated as follows:

$$
\text { Oscillation : } \omega(t)=\quad-A \sin (t), \quad 0 \leq t \leq T,
$$




$$
\text { Sculling : } \omega(t)= \begin{cases}P_{1}(t), & 0 \leq t \leq t_{1} ; \\ -\omega_{r}, & t_{1}<t \leq T_{r}-t_{1} ; \\ P_{2}\left(t-T_{r}\right), & T_{r}-t_{1}<t \leq T_{r}+\beta t_{1} ; \\ \omega_{p}, & T_{r}+\beta t_{1}<t \leq T-\beta t_{1} ; \\ P_{3}(t-T), & T-\beta t_{1}<t \leq T,\end{cases}
$$

$$
\begin{array}{lll}
P_{1}(t) & =-2 \omega_{r} \frac{t}{t_{1}}+2 \omega_{r} \frac{t^{3}}{t_{1}^{3}}-\omega_{r} \frac{t^{4}}{t_{1}^{4}}, & 0 \leq t \leq t_{1} ; \\
P_{2}\left(t-T_{r}\right)=2 \omega_{r} \frac{t-T_{r}}{t_{1}}-2 \omega_{r} \frac{\left(t-T_{r}\right)^{3}}{t_{1}^{3}}-\omega_{r} \frac{\left(t-T_{r}\right)^{4}}{t_{1}^{4}}, & T_{r}-t_{1}<t \leq T_{r} ; \\
P_{2}\left(t-T_{r}\right)=2 \omega_{r} \frac{t-T_{r}}{t_{1}}-2 \omega_{r}^{3} \frac{\left(t-T_{r}\right)^{3}}{\omega_{p}^{2} t_{1}^{3}}+\omega_{r}^{4} \frac{\left(t-T_{r}\right)^{4}}{\omega_{p}^{3} t_{1}^{4}}, & T_{r}<t \leq T_{r}+\beta t_{1} ; \\
P_{3}(t-T) & =-2 \omega_{r} \frac{t-T}{t_{1}}+2 \omega_{r}^{3} \frac{(t-T)^{3}}{\omega_{p}^{2} t_{1}^{3}}+\omega_{r}^{4} \frac{(t-T)^{4}}{\omega_{p}^{3} t_{1}^{4}}, & T-\beta t_{1}<t \leq T .
\end{array}
$$

In addition to purely sinusoidal and sculling rotations of the arm, we investigate the effect of prescribed arm deformations $[39,40]$ in the form of traveling-wave undulations, which are in synchrony with the corresponding rotation frequencies (Figures 2c, 2d). The time-dependent deformation is imposed in a single direction (in the $x$-axis), that is, vertically to the longitudinal axis of the arm, according to the following function

$$
d(z, t)=m z \sin \left(2 \pi w z-2 \pi f_{\{r, p\}} t+2 \pi \chi\{r, p\}\right),
$$


the arm. For both the sinusoidal oscillation and sculling motions, the values of $m=0.1$ and $w=0.05$ were assumed.

$f_{\{r, p\}}$ is the frequency of the traveling wave during the recovery and power strokes, respectively, and $\chi_{\{r, p\}}$ is the phase shift for the recovery and power strokes, respectively.

$$
\begin{array}{ccc}
\text { For oscillation + undulation: } & f_{r}=f_{p}=\frac{1}{T}, & \chi_{r}=\chi_{p}=0 . \\
\text { For sculling + undulation: } & f_{r}=\frac{1}{2 T_{r}}, f_{p}=\frac{1}{2 T_{p}}, & \chi_{r}=\frac{T_{r}-2 T_{p}}{4 T_{r}}, \chi_{p}=0.25-\frac{T_{r}}{2 T_{p}} .
\end{array}
$$

It is noted, that as a result of the unidirectional deformation, the arm elongates negligibly during the cyclic motion, and the tip acquires slightly sharper edges than when undeformed (Figures 2e, 2f).

All numerical solutions are initiated with an undeformed arm, at rest. A sigmoid function was applied to the deformation $d(z, t)$ only for the first few time steps of the $1^{r s t}$ cycle, in order to create small increments of the deformation displacement and facilitate the simulation. The expression assumed for the sigmoid was as follows

$$
S(t, a, b)=\frac{1}{1+e^{-a(t-b)}}
$$

where $a$ represents the growth rate and $b$ sets the displacement of the sigmoid function along the arm's length. For both sinusoidal oscillation and sculling, the values of $a=9$ and $b=0.9$ were chosen. $S(t, a, b)$ was equal to 1 for $t>0.25 T$, during sinusoidal oscillation, and for $t>T_{r}$, during sculling.

Wake vortex topology in the simulations was visualized by the $\lambda_{2}$ criterion, as proposed by Jeong and Hussain (1995) [41], according to which, a vortex core is located at regions where the second eigenvalue $\lambda_{2}$ of the $S^{2}+\Omega^{2}$ tensor is negative ( $\mathrm{S}$ and $\Omega$ correspond, respectively, to the symmetric and antisymmetric parts of the velocity gradient). The helicity, $H$, was also used for the identification of the vortical structures. Helicity is a measure of the angle between the vectors of velocity, $\mathbf{u}$, and vorticity, $\omega=\nabla \times \mathbf{u}$, defined as $H=\int \mathbf{u} \cdot \omega d V$ [42] in volume $V$, and describes the topological linking and direction of rotation of vortex lines [43, 44]. 


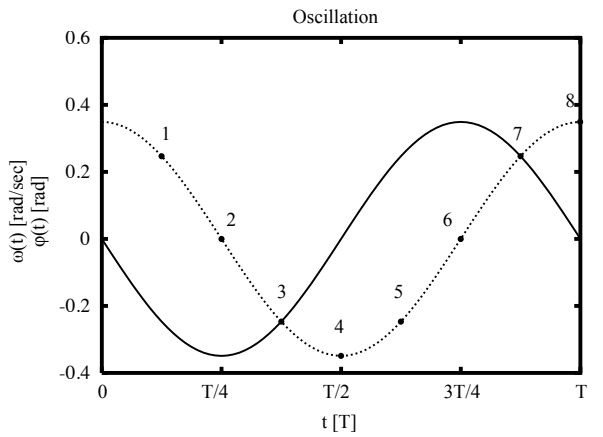

(a) - Angular Velocity

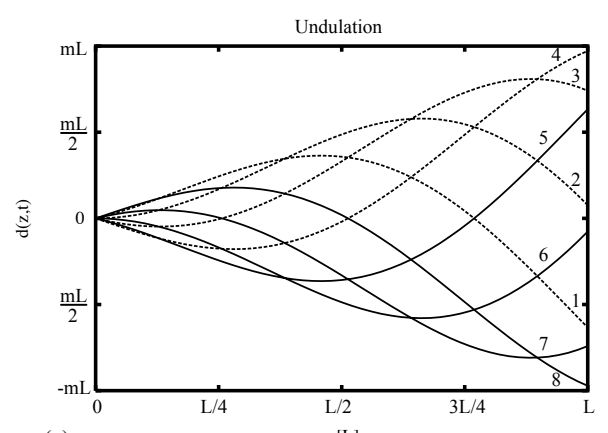

(c)

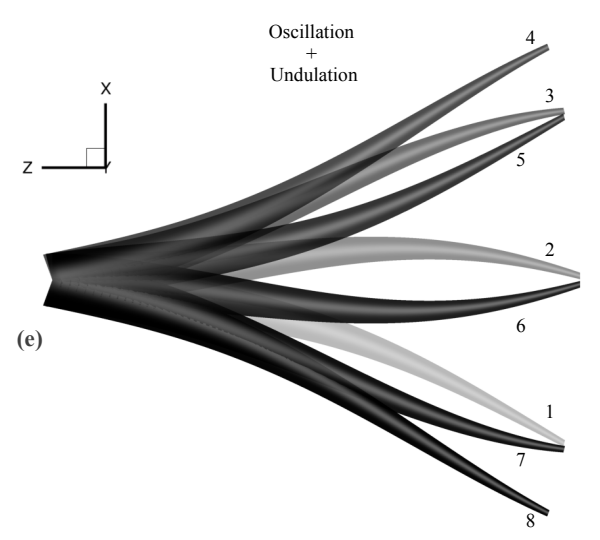

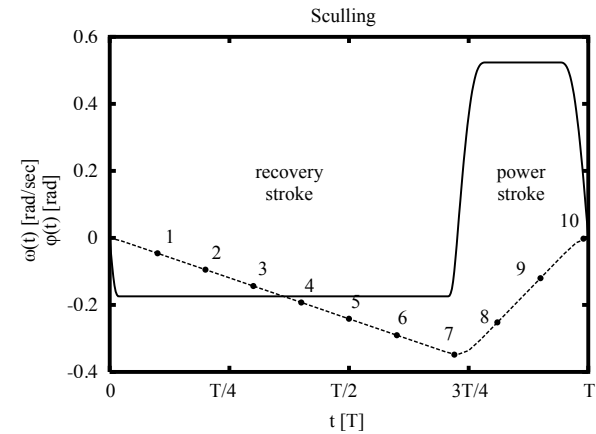

(b) - Angular Velocity _-.- Angle of rotation

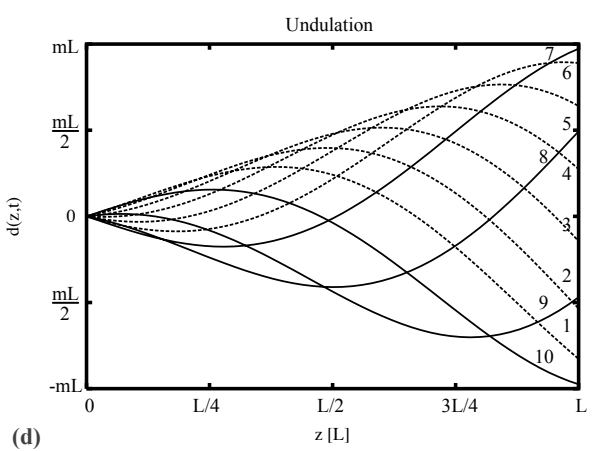

(d)

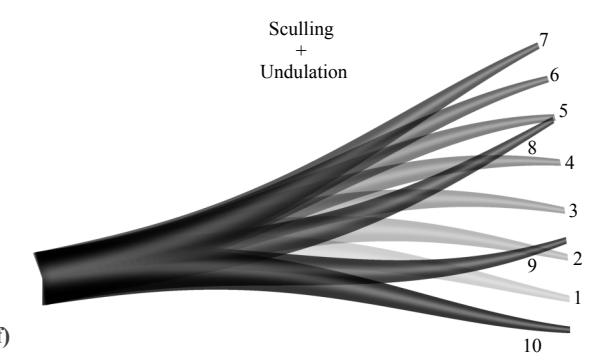

Figure 2: (a) Sinusoidal oscillation and (b) sculling motion profiles, displaying temporal variation of angular velocity and angle of rotation, for each case. (c-d) Time-dependent spatial deformation prescribed on the arm in the form of wavelike undulations that match up with the corresponding frequencies of oscillation and sculling.

(e) Oscillatory rotation around the arm base combined with wavelike arm undulation of the same frequency $\left(A=20^{\circ}\right)$. (f) Sculling rotation around the arm base combined with arm undulation in synchronized sculling frequencies, using the profiles shown in (b) and (d) $\left(A=10^{\circ}\right)$. Numerals are distinguished in left and right columns: (a), (c), (e), and (b), (d), (f). (L is the arm length; $m$ is the scaled amplitude, cf. Equation 4.) 


\subsection{Governing equations and numerical approaches}

A Newtonian fluid was assumed, governed by the incompressible Navier-Stokes equations [45, 46], according to the following formulation

$$
\begin{array}{r}
\frac{\partial \mathbf{u}}{\partial t}+(\mathbf{u} \cdot \nabla) \mathbf{u}=-\frac{\mathbf{1}}{\rho} \nabla \mathbf{p}+\nu \nabla^{2} \mathbf{u}, \\
\nabla \cdot \mathbf{u}=\mathbf{0},
\end{array}
$$

where $\mathbf{u}=[\mathrm{u}, \mathrm{v}, \mathrm{w}]$ is the velocity vector, $\rho$ is the fluid density, $\mathrm{p}$ is the pressure, and $v$ is the kinematic viscosity. The Reynolds number, $R e_{D}=U D / v$, is a characteristic of the flow, where $U$ is a velocity scale, and $D$ is, here, the base diameter of the octopus-like arm. Typical values of the Reynolds number for motions of octopus arms in seawater are of the order of $1000[47,48]$. This value $\left(R e_{D}=1000\right)$ was therefore adopted also in the current simulations, for a Reynolds number based on the arm base diameter [49, 14].

Two numerical approaches were used initially (Figures 3,4) to determine the mesh requirements that must be employed for sufficiently capturing the boundary layer in the investigated forced motions: a finite volume (FV) and an immersed boundary (IB) approach. The rest of the computations (Figures 5 - 11) were performed with the IB approach.

Finite Volume $(F V)$ framework: The FV numerical simulations were carried out with Fluent (ANSYS, Canonsburg, PA) by utilizing a second-order upwind discretization scheme for momentum, a second-order pressure discretization scheme and the SIMPLE algorithm for pressure-velocity coupling. The ANSA (BETA CAE Systems S.A., http://www.beta-cae.gr) mesh generation package was used to construct body-fitted prismatic elements at the near wall region of the arm within unstructured hybrid-type meshes. The spatial discretization around the arm was found more than sufficient to numerically capture the boundary layer growth and vortex generation during the arm movement, enabling accurate evaluation of viscous flow effects. A volume mesh of $18.76 \times 10^{6}$ tetrahedral elements was constructed in a computational domain that was 46D in the $x$-direction, 31D in the $y$-direction and 20D in the $z$-direction; the arm base was located 15D away from the upstream and lateral edges of the domain. The entire mesh rotated as a solid body without deformation and a moving mesh strategy, available in the flow solver, was adopted, allowing the 
high-resolution computational mesh to follow the arm at all instances of the cyclic movement. The quality and density of the mesh in the near-wake field, guarantees high resolution of the near-wall region and good preservation of the generated vortex patterns, with little diffusion, thus ensuring high fidelity and accuracy in our computations [14].

Immersed Boundary (IB) framework: The IB solution was computed using the curvilinear/immersed boundary (CURVIB) method, described in detail in Gilmanov and Sotiropoulos (2005) [24] and Ge and Sotiropoulos (2007) [25]. The code uses an immersed-boundary approach to solve the time-dependent, incompressible Navier-Stokes equations in generalized curvilinear coordinates. The fluid domain is discretized with a background curvilinear fixed grid, while the immersed body is treated as a sharp-interface boundary, discretized with an unstructured mesh. The position of the immersed body is tracked and its motion is accounted for by reconstructing the solution at the IB nodes in the immediate vicinity of the fluid/solid interface, using a quadratic interpolation (2nd order accurate) [24]. Nodes inside the solid immersed body (solid nodes) are blanked out and do not affect the solution on the fluid nodes. Pressure-field boundary conditions at the IB nodes are not explicitly required because of the hybrid formulation of the method [24]. Discretization in time is performed implicitly via a second-order accurate fractional step method for the time integration of the governing equations.

The CURVIB method is coupled with the matrix-free Newton-Krylov solver, available in the PETSc library, for the implicit solution of the momentum equations, and a very robust, multigrid-preconditioned GMRES solver, for the solution of the pressure-correction equation [25]. The flow solver is parallelized using the MPI and PETSc libraries. The background fluid mesh is parallelized by assigning a set of grid nodes to each processor using the PETSc's modules, which also manage parallel communications for structured mesh problems. The walltime per one step of the simulations with the CURVIB method (e.g. for those of Section 3.4), was 0.019 hrs, for a run of 800 steps per one cycle. The CURVIB method has been extensively validated and applied to a range of problems [50], e.g. vortexinduced vibrations; pulsatile, physiological, transitional flow in mechanical heart valves; aquatic swimming [12, 51]; turbulent flow in rivers with hydraulic structures; and flows past wind turbine rotors.

In this study, a 20 million structured cuboid grid was used for the fluid domain of the case solved with the IB method. The grid extended 30D in both the $x$ - and $z$-directions and 15D in the $y$-direction. A second, 1.5times smaller domain in the $z$-direction was constructed with 13 million structured elements to run the preliminary 

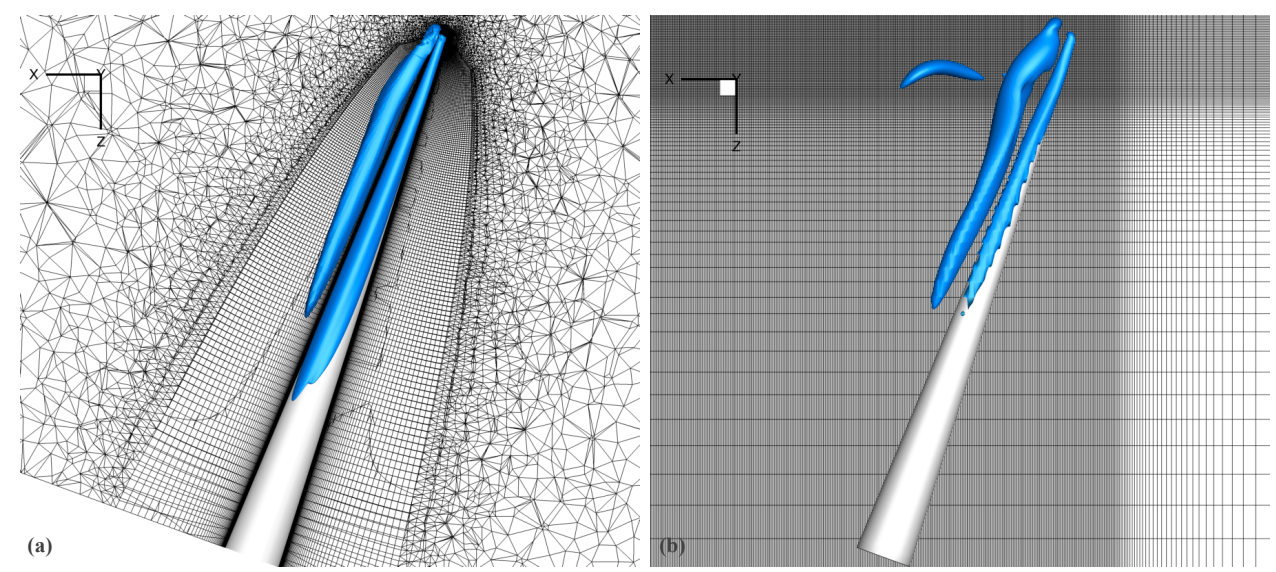

Figure 3: (a) Finite-volume solution, and (b) immersed-boundary solution of an octopus-like arm performing sinusoidal oscillation in the xz-plane. Instantaneous iso-contours of the $\lambda_{2}$ criterion [41] showing vortex topology $\left(\lambda_{2}=\right.$ $-5)$.

comparative studies of Figures 3a, 3b. In both grids, an inner region of size 8D x D x 2D was constructed with a uniform mesh, with an element spacing of $h=0.02 D$, to enclose the arm tip at all instances during the cyclic motions and to sufficiently capture the fine details of tip-initiated flow separation. The remaining grid was stretched towards this inner region with the use of a hyperbolic tangent stretching function. The aspect ratio of the largest corner element was 1.56.

The total hydrodynamic force, $F$, acting on the moving arm can be decomposed into the three Cartesian components $(F x, F y, F z)$, along the $x, y$ and $z$ directions of the computational domain. The hydrodynamic force coefficients $\left(C_{F x}, C_{F y}, C_{F z}\right)$ are defined as follows:

$$
C_{F i}=\frac{F_{i}}{\frac{1}{2} \rho U^{2} A}
$$

where $i=\{x, y, z\}$ and $A$ is the reference area, here taken as the projected frontal area of the undeformed arm.

\section{Results}

\subsection{Results of sinusoidal oscillation of the arm}

A solid-body sinusoidal oscillation was examined first with both the FV and IB numerical approaches, to verify the mesh requirements for sufficiently capturing the boundary layer growth around the arm. The arm rotated around 

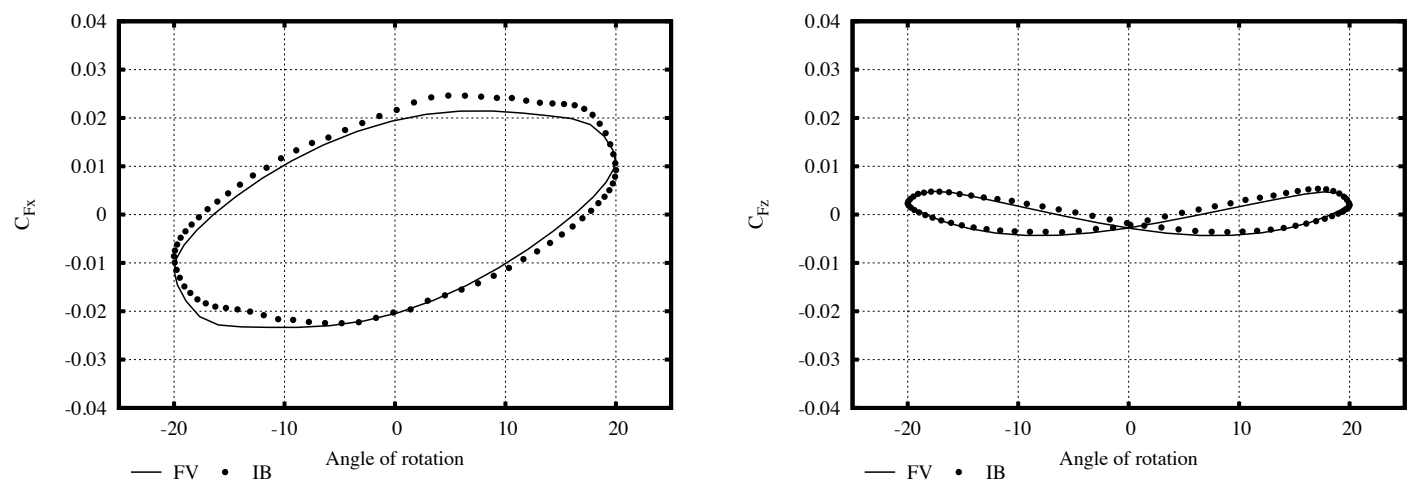

Figure 4: Comparison of the hydrodynamic force coefficients calculated by two numerical approaches, for an arm performing sinusoidal oscillation in the xz-plane. The solid line is evaluated by the Finite Volume (FV) method, whereas the dotted line by the Immersed Boundary (IB) method.

its base with a time-varying angular velocity $\omega(t)$ according to Equation 1, with $A=20^{\circ}$ (figure 3). Although small in magnitude, the motion resulted in two counter-rotating vortices on the trailing sides of the arm, for each stroke. Figure 3 shows vortex topology at the end of the second cycle, visualized as instantaneous iso-contours of the $\lambda_{2}$ criterion (according to Jeong and Hussain, 1995 [41]). The result of Figure 3a is calculated by the finite-volume method, as described in Section 2.3, and that of Figure 3b, by the immersed-boundary method, on the somewhat coarser mesh (13 million elements, cf. Section 2.3). In the FV case, the computational mesh rotated together with the arm, whereas, in the IB case, the background grid was fixed. The presence of two counter-rotating vortices originating from the tip and extending along the lee side of the arm, at a close distance from the wall, up to about half the arm length, was confirmed in both solutions.

The $\mathrm{C}_{F x}$ and $\mathrm{C}_{F z}$ force coefficients are displayed in Figures $4 \mathrm{a}, 4 \mathrm{~b}$, respectively, as a function of the angle of rotation. Time-periodicity was achieved after about 2 periods. The results show that the force components varied with the angle of rotation: for $\mathrm{C}_{F z}$, the motion produced positive thrust during upstroke and negative thrust during downstroke, resulting in an effectively almost zero forward thrust in the positive $z$-direction (integral of the $\mathrm{C}_{F z}$ curve with respect to the angle of rotation for one complete period). The solutions obtained from both the finite-volume and the immersed-boundary approaches are in good agreement.

Figures 5a, 5c display instantaneous vortex structures, as obtained with the IB approach, from later times of the 
cyclic motion at maximum amplitudes, using the finer grid (20 million elements, cf. Section 2.3). During most of this solid-body sinusoidal oscillation, the angular frequency is small, reaching maximum values only for a few instances. In addition, the amplitude and time duration of the motion are relatively large, allowing the vortices to diffuse between recurrent upstrokes and downstrokes of the arm. Nevertheless, few seemingly non-canonical vortical structures appear in the wake of the arm for both time instances presented.

\subsection{Results of sinusoidal arm oscillation combined with arm undulation}

The sinusoidal rotation of the arm (motion profile of Figure 2a) was subsequently combined with a wavelike arm undulation, according to Equation 4 and the parameters described in Section 2.2. The time-dependent spatial deformation along the arm's length is illustrated individually in Figure 2c, in which the dashed lines correspond to the upstroke (numerals from 1 to 4 ) and the solid lines to the downstroke (numerals from 5 to 8 ). The arm undulation is adjusted to be in synchrony with the sinusoidal rotation of the arm; therefore, the arm acquires maximum deformation at the maximum angle of rotation. As Figure 2e shows, incorporation of the undulation to the sinusoidal rotation, produces considerable deformation to the arm during the cyclic motion. The figure shows the deformed arm at instances where the activation function $d(z, t)$ is in full effect along the entire arm. Arm positions colored with light gray correspond to the upstroke (numerals 1 to 4), whereas darker-colored positions correspond to the downstroke (numerals 5 to 8 ). These positions can be tracked also in Figures 2a and $2 \mathrm{c}$ at the respective instances.

Figures $5 \mathrm{~b}$ and $5 \mathrm{~d}$ show vortex topology at positions of maximum arm deformation (and rotation). (It is noted that the $x z$-plane is slightly rotated for better visualization of the vortical structures near the tip.) Two counter-rotating vortices appear again in the lee side of the arm with respect to the direction of motion (upstroke and downstroke), although they are deformed, following the deformation of the arm wall. These figures also display an increased separation of flow from the tip, as compared to the undeformed arm at the same time instances (Figures 5a, 5c). There appears to be greater flow disturbance in the region near the tip's leeward side, shedding an extended street of non-canonical vortices in the wake, of opposite helicity. The increase in the flow separation from the tip may admittedly be enhanced by the unidirectional deformation that slightly distorts the tip. Nevertheless, the deformation itself increases the angle of attack for a bigger section of the arm near the tip, encouraging the disturbance of flow during both upstroke and downstroke. 

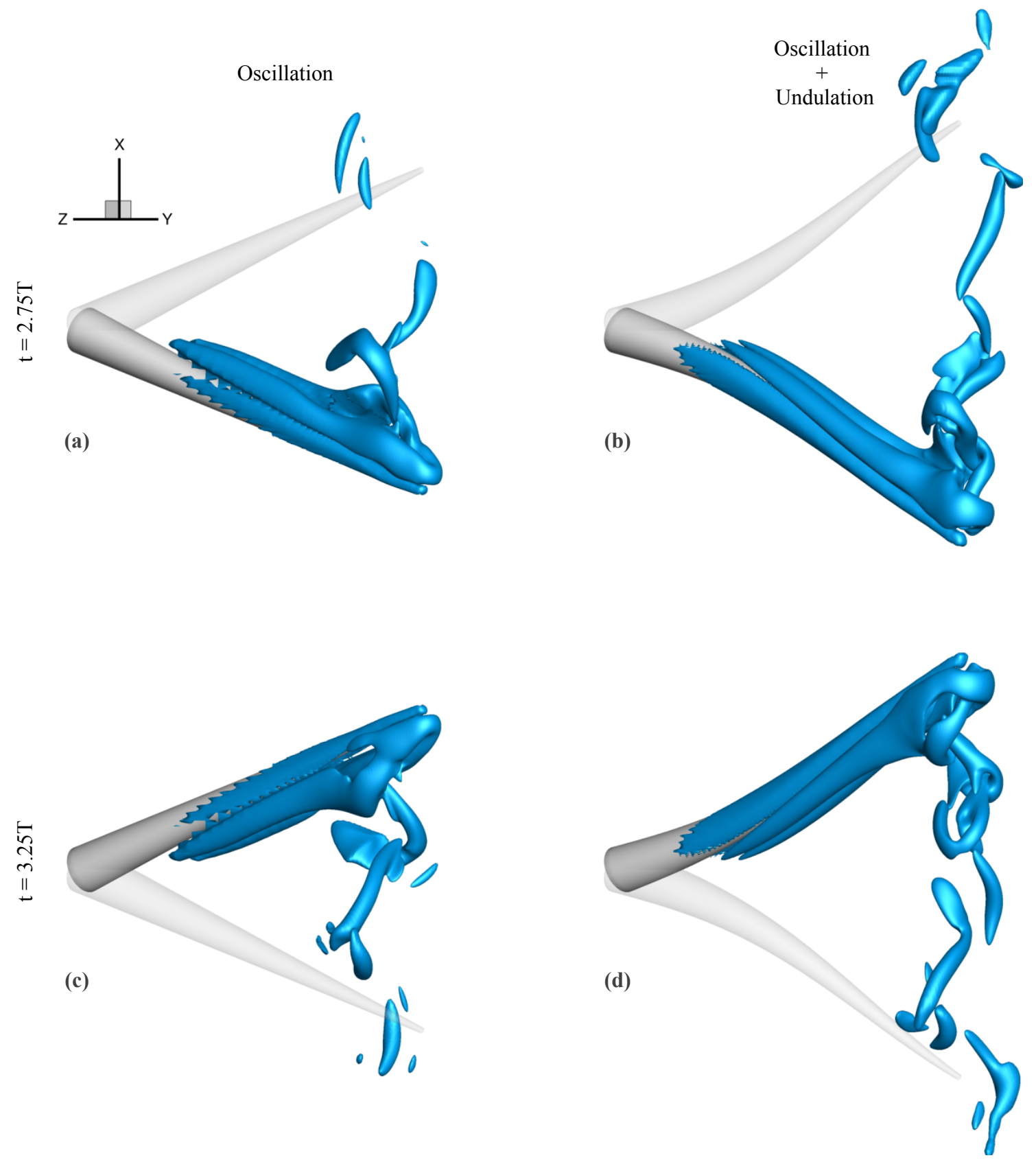

Figure 5: Instantaneous near-wake vortex patterns for sinusoidal oscillation around the arm base (a, c) without and (b, d) with arm undulations, obtained with the IB approach. Instances are shown at positions of maximum amplitude (a-b) $t=2.75 T$ and (c-d) $t=3.25 T\left(\lambda_{2}=-1\right)$. Positions at the opposite maximum amplitude are also depicted with light color, to indicate the direction of movement and the angular span. Note that the $x z$-plane is rotated for a better visualization of the vortical structures. 

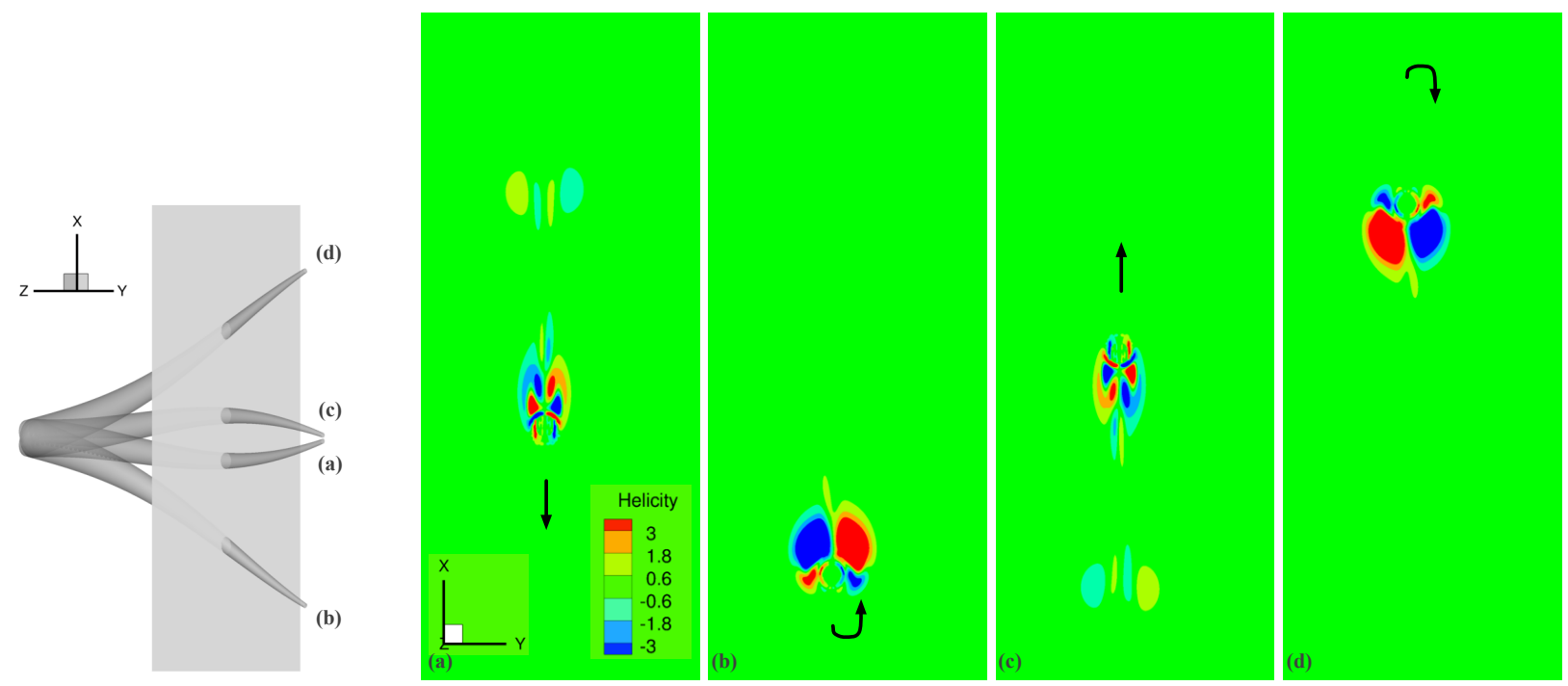

Figure 6: Evolution of vortical structures visualized according to helicity, on a fixed plane near the middle of the arm, at a distance $0.67 \mathrm{~L}$ from the arm base, for sinusoidal oscillation combined with arm undulation. (Left) Positions of the arm with respect to the plane. (Right) Helicity contours at $t=$ (a) $1.35 T$ (middle of upstroke, recovery), (b) $1.725 T$ (end of upstroke, recovery), (c) $1.85 T$ (middle of downtroke, power), (d) $2 T$ (end of downstroke, power). Instances in (b) and (d) correspond to instances shown in Figures 5b and 5d, respectively. Arrows show direction of movement.

Figures 6 and 7 display the evolution of vortical structures visualized according to helicity, on fixed planes near the middle of the arm, at a distance $0.67 L$ from the arm base, and the tip of the arm, at a distance $0.93 L$ from the arm base, respectively. There is considerable flow separation from the arm tip throughout the cyclic motion. Near the middle of the arm, the evolution of the flow is different. The well-formed counter-rotating vortices appear to be the main contributors to the generated thrust. These results are in consistent with the $\lambda_{2}$ vortex topology of Figures $5 \mathrm{~b}$ and $5 \mathrm{~d}$.

\subsection{Results of arm sculling}

A second cyclic motion was investigated, termed as sculling [52,35,36,37], by adopting the motion profile of Figure 2b. According to Equation 2 and the parameters described in Section 2.2, the motion is composed of a slow recovery (upstroke) and fast power (downstroke). Here, the following values were assumed: $A=10^{\circ} \omega_{r}=10^{\circ} / \mathrm{sec}$ and $\beta=3$. This motion profile was prescribed by a set of polynomial expressions $P_{1}, P_{2}, P_{3}$, according to Equations 2 and 3, to allow $C^{2}$ continuous transitions between the constant values of $\omega_{r}$ and $\beta \omega_{r}$ of the sculling. The solid-body 

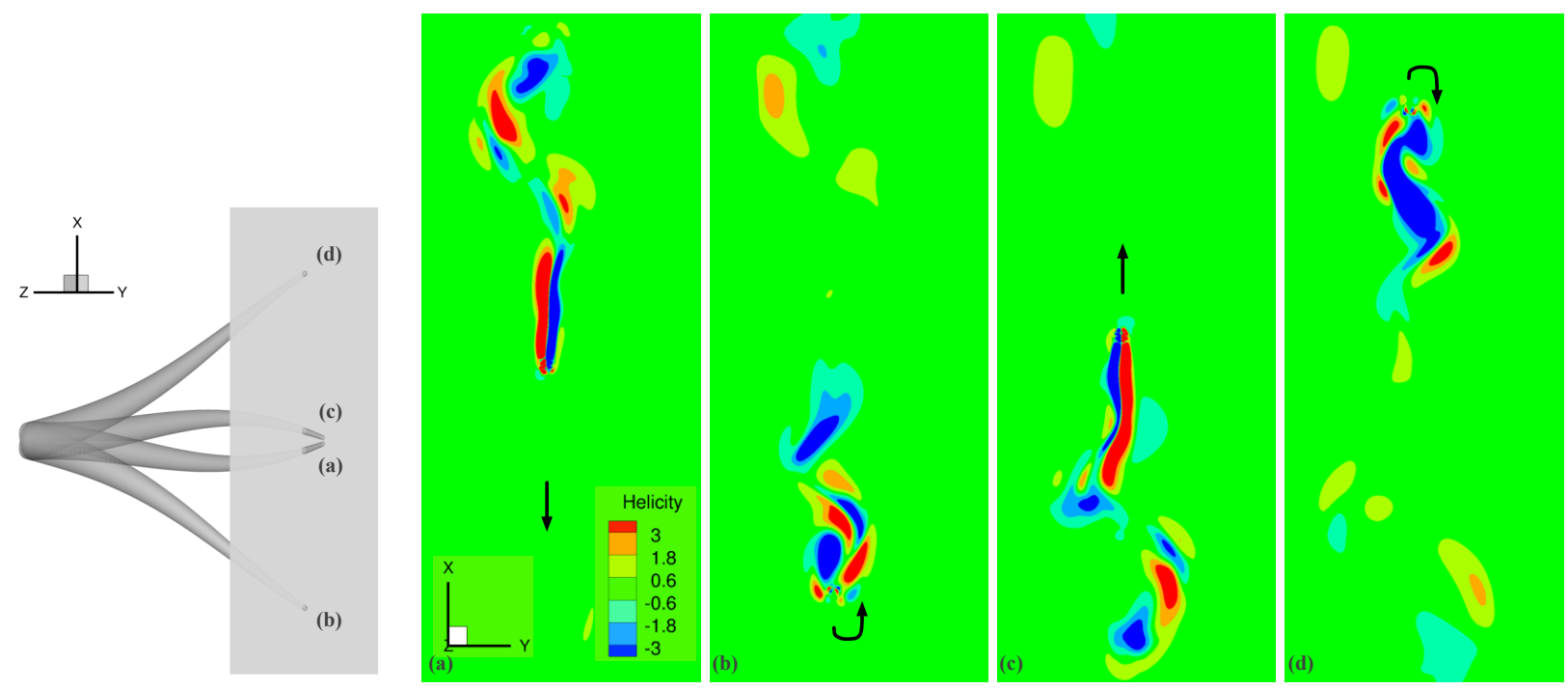

Figure 7: Evolution of vortical structures visualized according to helicity, on a fixed plane near the tip of the arm, at a distance $0.93 \mathrm{~L}$ from the arm base, for sinusoidal oscillation combined with arm undulation. (Left) Positions of the arm with respect to the plane. (Right) Helicity contours at $t=$ (a) $1.35 T$ (middle of upstroke, recovery), (b) $1.725 T$ (end of upstroke, recovery), (c) $1.85 T$ (middle of downtroke, power), (d) $2 T$ (end of downstroke, power). Instances in (b) and (d) correspond to instances shown in Figures 5b and 5d, respectively. Arrows show direction of movement.

motion examined here involves a smaller angular span than the sinusoidal oscillation described earlier.

Figure 8a depicts instantaneous vortical patterns as observed at the end of the recovery stroke (at $\mathrm{t}=1.725 \mathrm{~T})$ and Figure $8 \mathrm{c}$ at the end of the power stroke $($ at $\mathrm{t}=2 \mathrm{~T})$. Much like in the sinusoidal oscillation, the two counter-rotating vortices at the lee side of the arm are formed during this motion too; however, they appear thinner and somewhat more detached from the arm's wall. The angular frequency has considerably large values for the duration of the sculling movement, taking the value of $\omega_{r}$ for the most part of the cycle and then reaching the maximum value of $\beta \omega_{r}$ for almost one quarter of the cycle. In addition, the amplitude and time duration of the overall motion are relatively small (high $f_{r}$ and $f_{p}$ frequencies). Therefore, vortical structures generated in previous cycles do not diffuse in time for the subsequent upstroke and downstroke, and they continue to be visible in the flow field (vortical structures seen below the arm). The speed of the motion appears to result in abrupt separation from the tip, with very little shedding in the wake (as compared to the non-canonical vortices being shed during the slow sinusoidal oscillation, cf. Paragraph 3.1). 


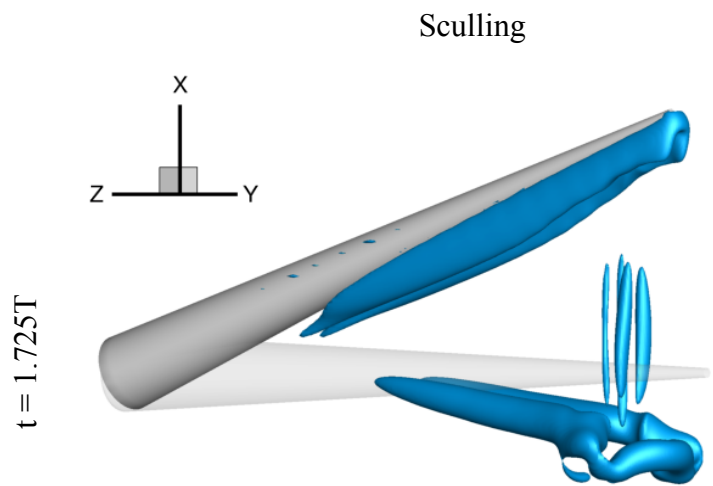

(a)

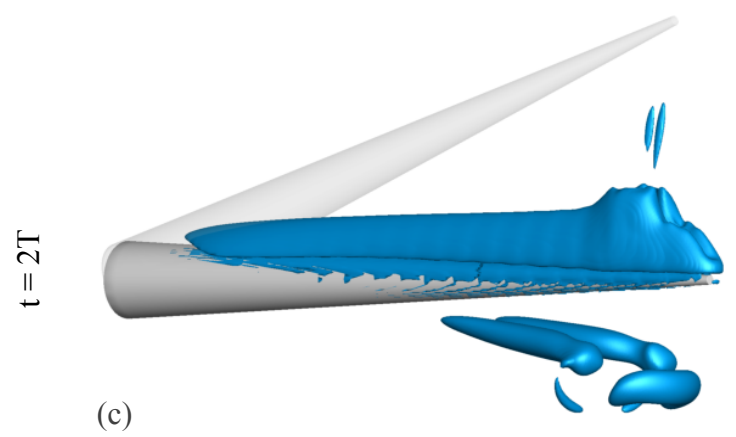

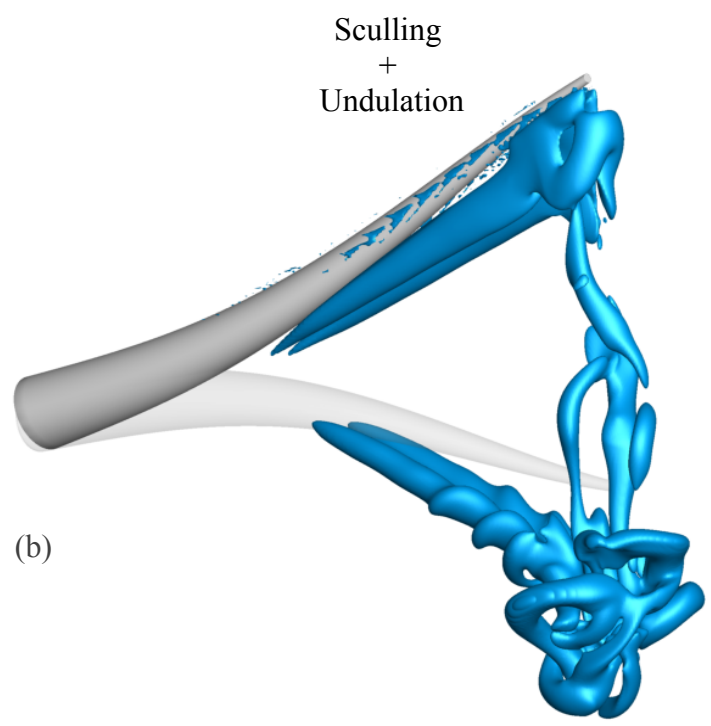

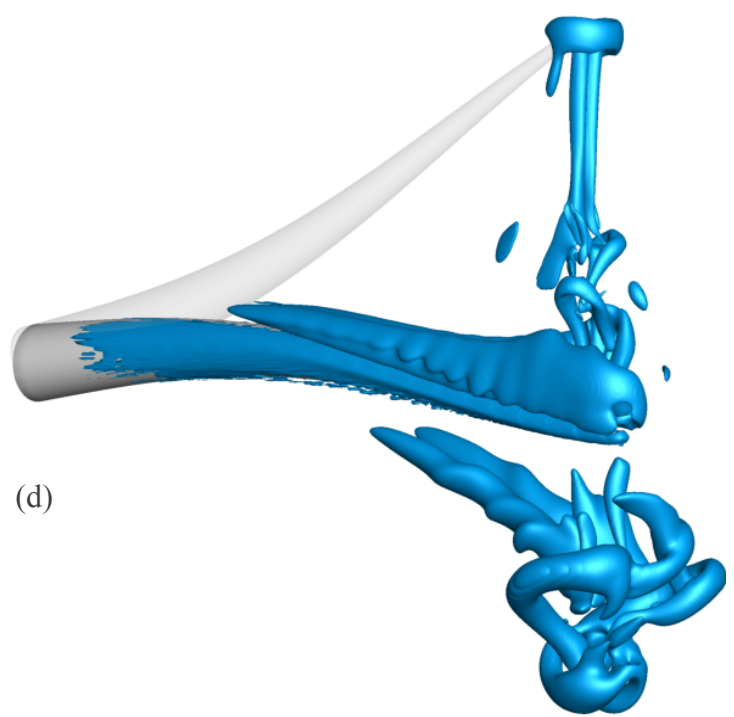

Figure 8: Instantaneous near-wake vortex patterns for sculling rotation around the arm base (a, c) without and (b, d) with arm undulations, obtained with the IB approach. Instances are shown at positions of maximum amplitude (a-b) $t=1.725$ and (c-d) $t=2 T\left(\lambda_{2}=-1\right)$. Positions at the opposite maximum amplitude are also depicted with light color, to indicate the direction of movement and the angular span. Note that the $x z$-plane is rotated for better visualization of the vortical structures.

\subsection{Results of arm sculling combined with arm undulation}

The sculling rotation of the arm (motion profile of Figure 2b) was combined with a wavelike arm undulation, according to Equation 4 and the parameters described in Section 2.2. The time-dependent spatial deformation along 

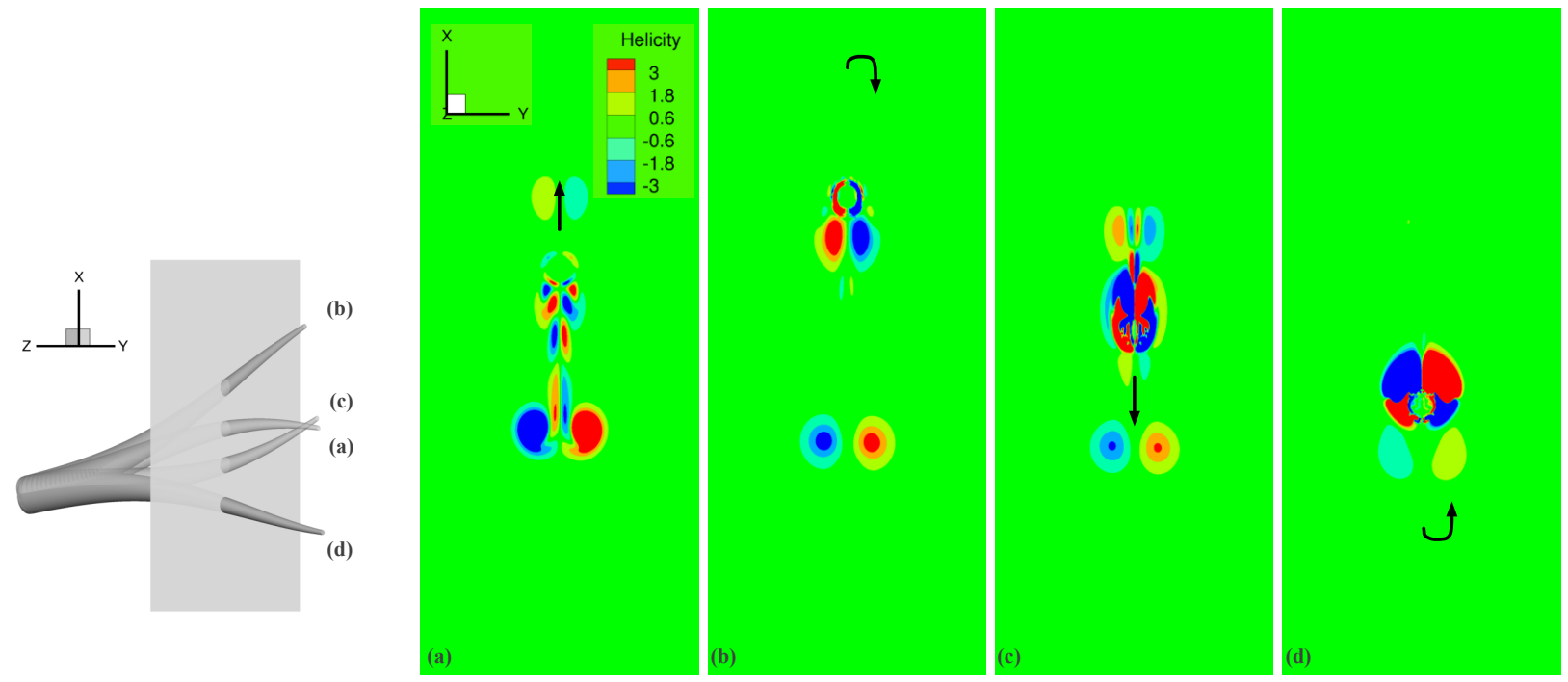

Figure 9: Evolution of vortical structures visualized according to helicity, on a fixed plane near the middle of the arm, at a distance $0.67 \mathrm{~L}$ from the arm base, for sculling combined with arm undulation. (Left) Positions of the arm with respect to the plane. (Right) Helicity contours at $t=$ (a) $2.5 T$ (middle of downstroke), (b) $2.75 T$ (end of downstroke), (c) $3 T$ (middle of ustroke), (d) $3.25 T$ (end of upstroke). Instances in (b) and (d) correspond to instances shown in Figures $8 \mathrm{~b}$ and $8 \mathrm{~d}$, respectively. Arrows show direction of movement.

the arm's length is illustrated individually in Figure 2d, in which the dashed lines correspond to the recovery stroke (upstroke, numerals from 1 to 7) and the solid lines to the power stroke (downstroke, numerals from 8 to 10). The fre-

quencies of the arm undulation during recovery and power strokes are tuned to match up with the sculling frequencies ( $f_{r}$ and $f_{p}$, respectively), so that maximum arm deformation is acquired at the end of each stroke. Figure $2 \mathrm{f}$ illustrates the deformation to the arm during the sculling motion. The figure shows the deformed arm at instances where the activation function $d(z, t)$ is in full effect along the entire arm. Arm positions colored with light gray correspond to the recovery stroke (numerals 1 to 7 ), whereas darker-colored positions correspond to the power stroke (numerals 8 to 10). These positions can be tracked also in Figures $2 b$ and $2 d$ at the respective instances.

Figures $8 \mathrm{~b}$ and $8 \mathrm{~d}$ show vortex topology at positions of maximum arm deformation. (Note again that the $x z$-plane is slightly rotated for better visualization of the vortical structures near the tip.) Compared to the results of Figures 5b, $5 \mathrm{~d}$, the two counter-rotating vortices in the leeward side of the arm, with respect to the direction of motion (recovery and power strokes), appear to follow only loosely the deformation of the arm wall, and are more detached near the 

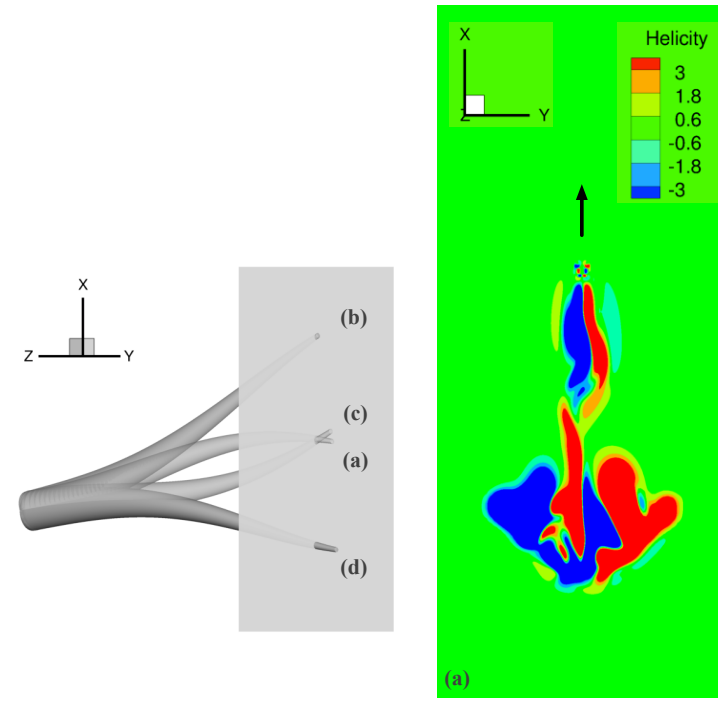
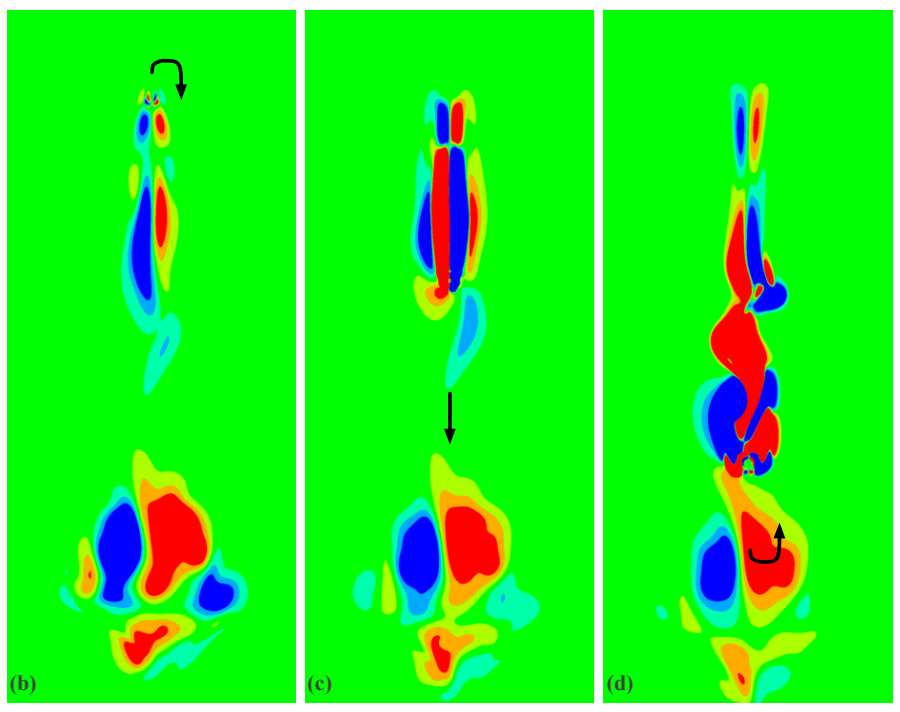

Figure 10: Evolution of vortical structures visualized according to helicity, on a fixed plane near the tip of the arm, at a distance $0.93 \mathrm{~L}$ from the arm base, for sculling combined with arm undulation. (Left) Positions of the arm with respect to the plane. (Right) Helicity contours at $t=$ (a) $2.5 T$ (middle of downstroke), (b) $2.75 T$ (end of downstroke), (c) $3 T$ (middle of ustroke), (d) $3.25 T$ (end of upstroke). Instances in (b) and (d) correspond to instances shown in Figures $8 \mathrm{~b}$ and $8 \mathrm{~d}$, respectively. Arrows show direction of movement.

tip. There is also increased separation of the flow from the tip and greater disturbance in the wake, in comparison to the undeformed arm at the same time instances (left column of Figures 8). Complex vortical structures generated in previous cycles do not appear to diffuse in time for the subsequent upstroke and downstroke. These structures are clearly visible in the time instances displayed in Figure 8 (right column): during the upward movement of the arm (Figure 8b), a small part of the large disturbed-flow structure generated in the previous cycle is drifted, while shedding new structures in the wake; during the the rapid downward return of the arm (like flapping, Figure 8d), the previous disturbed-flow structure is pushed further downwards.

Figures 9 and 10 display the evolution of vortical structures visualized according to helicity, on fixed planes near the middle of the arm, at a distance $0.67 \mathrm{~L}$ from the arm base, and the tip of the arm, at a distance $0.93 \mathrm{~L}$ from the arm base, respectively. There is extensive flow separation from the arm tip throughout the cyclic motion. Near the middle of the arm, however, the flow disturbance is different from that of the tip. The well-formed counter-rotating vortices seen at this region are the main generators of thrust. These results are in consistent with the $\lambda_{2}$ vortex topology of 


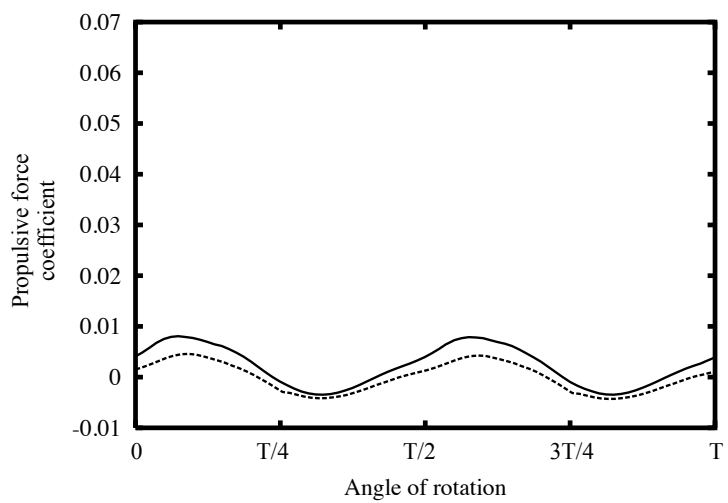

(a)

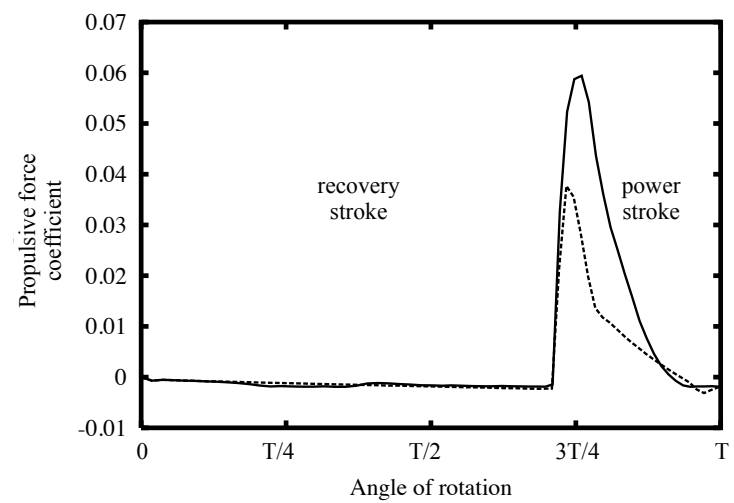

(b)

Figure 11: Temporal variation of the propulsive force coefficient, resulting from (a) oscillation and (b) sculling of the arm around its base, either alone or combined with arm undulations. Results obtained with the IB approach.

Figures $8 \mathrm{~b}$ and $8 \mathrm{~d}$.

\section{Discussion and conclusions}

The hydrodynamic propulsive force coefficients generated in the direction of positive $z$ (that is, towards the arm base) are shown for all four cases presented, in Figure 11. Calculated forces for pure sinusoidal oscillation and sculling are displayed with dashed lines, whereas those for rotations combined with undulations are depicted with solid lines, in both Figures 11a and 11b. The effect of arm undulation on the generated propulsive force is evident for both the sinusoidal oscillation and sculling. In sinusoidal oscillation, the increase is almost uniform, resulting in the generation of a small forward thrust (Figures 11a). In sculling, the effect is larger, particularly during the power stroke (Figures 11b), producing a considerably larger forward thrust.

Incorporation of arm deformation appears, thus, to increase the output thrust of the combined system. Indeed, a single arm rotating as a solid body around its base is a single-degree-of-freedom system, with a single joint at its base. This system adds some energy to the surrounding flow field in the form of the vortical structures seen in Figures 5a, $5 \mathrm{c}$ and $8 \mathrm{a}, 8 \mathrm{c}$. The prescribed wavelike undulations of the arm, however, increase drastically the number of degreesof-freedom of the system, since every finite element on the arm's surface can be thought to act as a finite joint, adding extra energy to the flow field (Figures $5 b, 5 d$ and $8 b, 8 d$ ); and, hence, producing extra thrust. This case differs from 
a compliant arm that would deform as a result of its interaction with the flow, and would require a fluid-structure interaction numerical approach to investigate.

We are interested specifically in the prescribed activation of the arm, that would be based on realistic octopus arm kinematics (such as that of Figure 1), since octopus appendages are known to actively take part in many of the observed behaviors (e.g., arm-swimming, head-up swimming, crawling, etc.). Admittedly, these movements are far more complex than the simplified motion profiles we considered here. We used the immersed boundary approach in order to simulate the deformation of an arm during these simplified movements and better understand the unsteady hydrodynamics of the mechanism, since the extremely large deformations of the mesh required with body-fitted finite-element or finite-volume approaches, are very hard to achieve even with the most advanced mesh deformation techniques. It was found that the sculling motion combined with arm undulation is an effective propulsive scheme for an octopus-like arm. One direction towards simulating more realistic locomotion patterns is to include translation of the arm as a result of the generated propulsive force. This aspect, combined with a more accurate definition of the deformation on the arm, may provide a deeper insight underlying cephalopod-like propulsive systems. Furthermore, the inclusion of a second or more arms may help analyze possible interaction among different arms.

Aiming at a computation tool able to model intense motions, as found in aquatic locomotion instances, two numerical methods were compared initially. The immersed boundary method demonstrates its suitability for handling such motions. We aim to further adjust this method for special flow characteristics of time-varying geometries under more complex prescribed motions.

\section{Acknowledgments}

This work was supported in part by the European Commission (EC) and the General Secretariat for Research and Technology (GSRT) of the Hellenic Ministry of Education via the ESF-GSRT HYDRO-ROB Project [PE7(281)] and the EC-ERDF BIOSYS-KRIPIS Project [MIS-448301 (2013SE01380036)]. Parts of these simulations were carried out on the CaSToRC High-Performance Computing (HPC) system, of the LINKSCEEM/Cy-Tera resources (Project pro14a108) and the PRACE Tier-1 HPC system (Project CepFlow, 12DECI0048). The authors would like to thank B. Hochner, T. Flash, M. Sfakiotakis, X. Zabulis, M. Kuba, J. Oikonomidis, A. Chatzidaki, Th. Evdaimon, and S. 
Stefanou, for their assistance with these studies.

\section{References}

[1] W.M. Kier, K.K. Smith, Tongues, tentacles and trunks: the biomechanics of movement in muscular-hydrostats, Zool J Linn Soc 83 (1985) $307-324$

[2] C.L. Huffard, Locomotion by abdopus aculeatus (cephalopoda: Octopodidae): Walking the line between primary and secondary defenses, J Exp Biol 209 (2006) 3697-3707.

[3] E.J. Anderson, M.E. Demont, The mechanics of locomotion in the squid loligo pealei: locomotory function and unsteady hydrodynamics of the jet and intramantle pressure, J Exp Biol 203 (2000) 2851-2863.

[4] M. Sfakiotakis, D.M. Lane, J.B.C. Davies, Review of fish swimming modes for aquatic locomotion, IEEE J Ocean Eng 24 (2) (1999) $237-252$.

[5] G.V. Lauder, E.G. Drucker, Forces, fishes, and fluids: Hydrodynamic mechanisms of aquatic locomotion, Physiology 17 (2002) $235-240$.

[6] J.C. Liao, D.N. Beal, G.V. Lauder, MS. Triantafyllou, Fish exploiting vortices decrease muscle activity, Science 302 (2003) $1566-1569$.

[7] J.C. Liao, D.N. Beal, G.V. Lauder, M.S. Triantafyllou, The kármán gait: novel body kinematics of rainbow trout swimming in a vortex street, J Exp Biol 206 (2003) 1059-1073.

[8] E.D. Tytell, G.V. Lauder, The hydrodynamics of eel swimming. i. wake structure, J Exp Biol 207 (2004) 1825-1841.

[9] E.D. Tytell, The hydrodynamics of eel swimming. ii. effect of swimming speed, J Exp Biol 207 (2004) 3265-3279.

[10] J.O. Dabiri, S.P. Colin, J.H. Costello, Fast-swimming hydromedusae exploit velar kinematics to form an optimal vortex wake, J Exp Biol 209 (2006) 2025-2033.

[11] I.K. Bartol, P.S. Krueger, J.T. Thompson, W.J. Stewart, Swimming dynamics and propulsive efficiency of squids throughout ontogeny, Integr Comp Biol 48 (6) (2008) 720-733.

[12] I. Borazjani, F. Sotiropoulos, Numerical investigation of the hydrodynamics of carangiform swimming in the transitional and inertial flow regimes, J Exp Biol 211 (2008) 1541-1558.

[13] G.V. Lauder, Animal locomotion, Heidelberg, Germany, Springer-Verlag, 2010, Ch. Swimming hydrodynamics: Ten questions and the technical approaches needed to resolve them, pp. 3-15.

[14] A. Kazakidi, V. Vavourakis, D. P. Tsakiris, J. Ekaterinaris, A numerical investigation of flow around octopus-like arms: near-wake vortex patterns and force development, Comp. Meth. Biomech. Biomed. Eng. 18 (12) (2015) 1321-1339.

[15] P.D. Thomas, C.K. Lombard, Geometric conservation law and its application to flow computations on moving grids, AIAA Journal 17 (10) (1979) 1030-1037.

[16] I. Demirdzic, M. Peric, Space conservation law in finite volume calculations of fluid flow, Int. J. Numer. Meth. Fluids 8 (1988) $1037-1050$.

[17] C. Peskin, Flow pattern around heart valves: a numerical method, J Comput Phys 10 (1972) 252-271.

[18] C. Peskin, D. McQueen, Shared-memory parallel vector implementation of the immersed boundary method for the computation of blood flow in the beating mammalian heart, J. Supercomput. 11 (1997) 213-236. 
[19] D. Goldstein, R. Handler, L. Sirovich, Modeling a no-slip flow boundary with an external force field, J. Comput. Phys. 105 (1993) $354-366$.

[20] R. Cortez, M. Minion, The blob projection method for immersed boundary problems, J. Comput. Phys. 161 (2000) $428-453$.

[21] R. LeVeque, Z. Li, Immersed interface method for stokes flow with elastic boundaries or surface tension, SIAM J. Sci. Comput. 18 (1997) $709-735$

[22] J. Mohd-Yusof, Combined immersed boundaries/b-splines methods for simulations of flows in complex geometries. center for turbulence research., Annual Research Briefs, Stanford University, NASA Ames (1997) 317-327.

[23] E. Fadlun, R. Verzicco, P. Orlandi, J. Mohd-Yusof, Combined immersed-boundary finite-difference methods for three-dimensional complex flow simulations, J. Comput. Phys. 161 (2000) 35-60.

[24] A. Gilmanov, F. Sotiropoulos, A hybrid cartesian/immersed boundary method for simulating flows with 3d, geometrically complex, moving bodies, J. Comp. Phys. 207 (2005) 457-492.

[25] L. Ge, F. Sotiropoulos, A numerical method for solving the 3d unsteady incompressible navier-stokes equations in curvilinear domains with complex immersed boundaries, J Comput Phys 225 (2007) 1782-1809.

[26] A. Kazakidi, D.P. Tsakiris, F. Sotiropoulos, J.A. Ekaterinaris, Numerical investigation of aquatic locomotion with cephalopod-like appendages, in: 19th Congr Europ Soc Biomech, (ESB2013), Patra, Greece, 2013.

[27] A. Kazakidi, D. P. Tsakiris, F. Sotiropoulos, J. A. Ekaterinaris, A computational fluid dynamic study of intense cephalopod-like motions, in: 44th AIAA Fluid Dynamics Conference, AIAA AVIATION, Atlanta, GA, USA, 2014.

[28] T.-H. Wu, R.-S. Guo, G.-W. He, Y.-M. Liu, D. Qi, Simulation of swimming of a flexible filament using the generalized lattice-spring latticeBoltzmann method, J Exp Biol 349 (2014) 1-11.

[29] A. Kazakidi, M. Kuba, A. Botvinnik, M. Sfakiotakis, T. Gutnick, S. Hanassy, G. Levy, J. A. Ekaterinaris, T. Flash, B. Hochner, D. P. Tsakiris, Swimming patterns of the Octopus vulgaris, in: 22nd Annual Meeting NCM Society, Venice, Italy, 2012.

[30] A. Kazakidi, S. Stefanou, X. Zabulis, M. Kuba, A. Botvinnik, S. Hanassy, M. Sfakiotakis, T. Gutnick, J. A. Ekaterinaris, T. Flash, B. Hochner, D. P. Tsakiris, Motion reconstruction of arm swimming in the Octopus vulgaris, in: 19th Congress of the European Society of Bioengineering (ESB), Patra, Greece, 2013.

[31] A. Kazakidi, S. Stefanou, X. Zabulis, M. Kuba, J. A. Ekaterinaris, T. Flash, B. Hochner, D. P. Tsakiris, 3d reconstruction of octopus arm swimming motion, in: 7th World Congress of Biomechanics (WCB), Boston, MA, USA, 2014.

[32] A. Kazakidi, X. Zabulis, D. P. Tsakiris, Vision-based 3d motion reconstruction of octopus arm swimming and comparison with an 8-arm underwater robot, in: IEEE Int. Conf. Rob. Autom. (ICRA'15), Seattle, WA, USA, 2015 in press.

[33] Y. Yekutieli, R. Mitelman, B. Hochner, T. Flash, Analyzing octopus movements using three-dimensional reconstruction, J. Neurophysiol. 98 (2007) 1775-1790.

[34] R. Y. Tsai, A versatile camera calibration technique for high-accuracy $3 \mathrm{~d}$ machine vision metrology using off-the-shelf TV cameras and lenses, IEEE Journal of Robotics and Automation 3 (4) (1987) 323-344.

[35] M. Sfakiotakis, A. Kazakidi, N. Pateromichelakis, D.P. Tsakiris, Octopus-inspired eight-arm robotic swimming by sculling movements, in: IEEE Int Conf Rob Autom, (ICRA 2013), Karlsruhe, Germany, 2013, pp. 5135-5141. 
[36] M. Sfakiotakis, A. Kazakidi, D.P. Tsakiris, Turning maneuvers of an octopus-inspired multi-arm robotic swimmer, in: 21st Med Conf Control Autom, (MED'13), Chania, Greece, 2013, pp. 1343-1349.

[37] M. Sfakiotakis, A. Kazakidi, A. Chatzidaki, T. Evdaimon, D. P. Tsakiris, Multi-arm robotic swimming with octopus-inspired compliant web, in: IEEE/RSJ Int. Conf. on Int. Rob. Syst. (IROS'14), Chicago, Illinois, USA, 2014, pp. 302-308.

[38] M. Sfakiotakis, A. Kazakidi, D. P. Tsakiris, Octopus-inspired multi-arm robotic swimming, Bioinspir. Biomim. (2015) in press.

[39] V. Vavourakis, D. Bampasakis, A. Kazakidi, N. Pateromichelakis, J.A. Ekaterinaris, D.P. Tsakiris, Generation of primitive behaviors for non-linear hyperelastic octopus-inspired robotic arm, in: IEEE Int Conf Biomed Robotics Biomechatron, (BioRob) Roma, Italy, 2012, pp. $725-730$

[40] V. Vavourakis, A. Kazakidi, D.P. Tsakiris, J.A. Ekaterinaris, A nonlinear dynamic finite element approach for simulating muscular hydrostats, Comput Methods Biomech Biomed Engin 17 (8) (2014) 917-931.

[41] J. Jeong, F. Hussain, On the identification of a vortex, J Fluid Mech 285 (1995) 69-94.

[42] H. Moffatt, A. Tsinober, Helicity in laminar and turbulent flow, Ann Review Fluid Mech 24 (1992) $281-312$.

[43] D. Degani, A. Seginer, Y. Levy, Graphical visualization of vortical flows by means of helicity, AIAA Journal 28 (8) (1990) $1347-1352$.

[44] A. Pobitzer, R. Peikert, R. Fuchs, B. Schindler, A. Kuhn, H. Theisel, K. Matkovic, H. Hauser, The state of the art in topology-based visualization of unsteady flow, Computer Graphics Forum 30 (6) (2011) 1789-1811.

[45] G.K. Batchelor, An introduction to fluid dynamics, Cambridge University Press, 2000.

[46] D.J. Tritton, Physical fluid dynamics, Oxford University Press, 1988.

[47] Y. Gutfreund, T. Flash, Y. Yarom, G. Fiorito, I. Segev, B. Hochner, Organization of octopus arm movements: A model system for studying the control of flexible arms, J Neurosc 16 (1996) 7297-7307.

[48] Y. Yekutieli, R. Sagiv-Zohar, R. Aharonov, Y. Engel, B. Hochner, T. Flash, Dynamic model of the octopus arm. i. biomechanics of the octopus reaching movement, J Neurophysiol 94 (2) (2005) 1443-1458.

[49] A. Kazakidi, V. Vavourakis, N. Pateromichelakis, J.A. Ekaterinaris, D.P. Tsakiris, Hydrodynamic analysis of octopus-like robotic arms, in: IEEE Int Conf Rob Autom, ICRA 2012, St Paul, Minnesota, USA, 2012, pp. 5295-5300.

[50] I. Borazjani, L. Ge, F. Sotiropoulos, Curvilinear immersed boundary method for simulating fluid structure interaction with complex $3 \mathrm{~d}$ rigid bodies, J Comput Phys 227 (2008) 7587-7620.

[51] I. Borazjani, F. Sotiropoulos, E. D. Tytell, G. V. Lauder, Hydrodynamics of the bluegill sunfish c-start escape response: three-dimensional simulations and comparison with experimental data, J Exp Biol 215 (2012) 671-684.

[52] M. Sfakiotakis, A. Kazakidi, N. Pateromichelakis, J.A. Ekaterinaris, D.P. Tsakiris, Robotic underwater propulsion inspired by the octopus multi-arm swimming, in: IEEE Int Conf Rob Autom, (ICRA 2012), St Paul, Minnesota, USA, 2012, pp. $3833-3839$. 